\title{
Roles of the superoxide dismutase SodB and the catalase KatA in the antibiotic resistance of Campylobacter jejuni
}

\author{
Sunyoung Hwang ${ }^{2}$, Sangryeol Ryu ${ }^{2}$ and Byeonghwa Jeon ${ }^{1}$ \\ The Journal of Antibiotics (2013) 66, 351-353; doi:10.1038/ja.2013.20; published online 3 April 2013
}

Keywords: antibiotic resistance; Campylobacter; oxidative stress resistance

Reactive oxygen species (ROS) are inevitable by-products from aerobiosis and give damages to biomolecules. ${ }^{1}$ Recently, it was reported that treatment with bactericidal antibiotics enhances the generation of ROS in Escherichia coli, and then the increased ROS production affects bacterial lethality under the treatment of bactericidal antibiotics. ${ }^{2}$ This is considered a new novel paradigm of bacterial killing by antibiotics but has thus far been examined only in a few bacterial species, including E. coli, Staphylococcus aureus and Enterococcus faecalis. ${ }^{2,3}$ In Listeria monocytogenes, however, oxidative stress mutations did not alter the lethal kinetics of bactericidal antibiotics. ${ }^{4}$ These previous reports suggest that the ROS-mediated lethality mechanism may have different impacts on antibiotic resistance depending on bacterial species.

Campylobacter species, particularly Campylobacter jejuni, are a major bacterial cause of human gastroenteritis, accounting for approximately 400-500 million infection cases in humans worldwide per year. ${ }^{5}$ In addition, increasing prevalence of Campylobacter resistant to clinically important antibiotics, such as fluoroquinolones and macrolides, emerged as a serious public health concern. ${ }^{6}$ C. jejuni is a microaerophilic bacterium and has unique oxidative stress defense systems. ${ }^{7}$ For example, $C$. jejuni possesses only the sole superoxide dismutase $(\operatorname{sod} B)$ and catalase $(\mathrm{kat} A)$ gene, ${ }^{7}$ whereas E. coli carries three superoxide dismutase genes ( $\operatorname{sod} A, \operatorname{sodB}$ and $\operatorname{sod} C)$ and two catalase genes $(k a t G$ and $k a t E) .{ }^{1}$ In this study, we constructed single mutants of $\operatorname{sodB}$ and katA, two key genes of oxidative stress resistance, and a double mutant of $\operatorname{sodB}$ and katA, and investigated the effect of oxidative stress defense systems on antibiotic resistance in C. jejuni.

C. jejuni NCTC 11168 and its derivatives were routinely grown at $42^{\circ} \mathrm{C}$ on Mueller-Hinton (MH) media (Difco, Franklin Lakes, NJ, USA) under microaerobic conditions generated by MART
(Anoxomat, Mart Microbiology B.V., Drachten, the Netherlands). Culture medium was supplemented with kanamycin $\left(50 \mathrm{mgl}^{-1}\right)$ or chloramphenicol $\left(10 \mathrm{mgl}^{-1}\right)$, where required. We knocked out the $\operatorname{sodB}$ and $k a t A$ genes with suicide plasmids as described previously. ${ }^{8}$ Briefly, a DNA fragment containing $\operatorname{sodB}$ and its flanking region was amplified from $C$. jejuni NCTC 11168 using the primers sodB_F: GAC TTA TAT CAA GGC TGG GAT TAT TGA and sodB_R: CAC TCC ATT TCC ACA ACC AAA ATC AAG T, and was ligated to pUC19. The chloramphenicol resistance cassette (cat) was PCR-amplified from pRY112, ${ }^{9}$ and inserted into a BglII site of $\operatorname{sodB}$ in pUC19. To construct a katA mutant, the PCR product amplified with the primers (katA_F: TAA CGA TTT TGG ATC CAT TAT AGC and katA_R: AAT TAT TTT TCT AAA GCT TTT TTA ATT CC) was cloned to pUC19. The kanamycin resistance cassette $(a p h A)$ was generated by PCR from pMW $10,{ }^{10}$ and inserted to a BglII site of katA on pUC19. The constructed suicide plasmids were introduced to $C$. jejuni NCTC 11168 by electroporation. The $\operatorname{sodB}$ and kat $A$ mutants were selected by growing on $\mathrm{MH}$ agar plates supplemented with chloramphenicol $\left(10 \mathrm{mgl}^{-1}\right)$ and kanamycin $\left(50 \mathrm{mgl}^{-1}\right)$, respectively. The sodB and katA double mutant was constructed by transferring the $\operatorname{sodB}$ mutation to the katA mutant by natural transformation. ${ }^{8}$ To construct complementation strains, intact copies of $\operatorname{sodB}$ and katA were integrated into the chromosome with a method described elsewhere. ${ }^{11}$ Briefly, DNA fragments containing the intact copy of sodB and katA were amplified with primer pairs (sodB-CF: GAT TAA ATA TCT AGA AAT TTT ACC CAC and sodB-CR: CCC TTT TTG TCT AGA ATG TTG AC and katA-CF: TTT TGA AAT TTA TTA TCT AGA AAA TGC AAT TAT TC and katA-CR: TTA TTA TAA TCT CTA GAT TGC CAC CAA AAG) and cloned into an XbaI site of a pUC19 derivative carrying an rRNA gene cluster. ${ }^{11-13}$ The plasmids were delivered to the $\operatorname{sodB}$ and katA mutants by electroporation. Allelic

${ }^{1}$ Department of Public Health Sciences, University of Alberta, Edmonton, Alberta, Canada and ${ }^{2}$ Department of Food and Animal Biotechnology, Department of Agricultural Biotechnology, and Center for Agricultural Biomaterials, Seoul National University, Seoul, Republic of Korea

Correspondence: Dr B Jeon, Department of Public Health Sciences, University of Alberta, Edmonton, Alberta T6G 2G7, Canada.

E-mail: bjeon@ualberta.ca

or Dr S Ryu, Department of Food and Animal Biotechnology, Department of Agricultural Biotechnology, and Center for Agricultural Biomaterials, Seoul National University, Seoul 151-921, Republic of Korea.

E-mail: sangryu@snu.ac.kr

Received 17 November 2012; revised 14 February 2013; accepted 18 February 2013; published online 3 April 2013 
exchanges in the sodB and katA mutants were confirmed by PCR and sequencing a flanking region of the mutated genes (data not shown). The minimum inhibitory concentrations (MICs) of several antibiotics (ciprofloxacin, erythromycin, cefotaxime, gentamicin, rifampicin, polymyxin B and tetracycline) were measured with the microtitre broth dilution method. ${ }^{14}$ Rapid killing of Campylobacter by antibiotics was determined as described previously with some minor modifications. ${ }^{9}$ Briefly, C. jejuni grown overnight on $\mathrm{MH}$ agar plates was resuspended in fresh $\mathrm{MH}$ broth to an $\mathrm{OD}$ at $600 \mathrm{~nm}$ of $\sim 0.06\left(10^{7} \mathrm{CFU} \mathrm{ml}{ }^{-1}\right)$. Aliquots of bacterial suspension were exposed to antibiotics. At every $1 \mathrm{~h}$, samples were serially diluted and applied to $\mathrm{MH}$ agar plates for viable counts.

The katA mutation decreased the MICs of ciprofloxacin and rifampicin by two-fold compared with the wild-type. The MIC reduction in ciprofloxacin was restored by complementation, while that of rifampicin was not complemented (data not shown). Although the sodB single mutation did not make any differences in the MICs of the tested antibiotics, the $\operatorname{sodB}$ and katA double mutation further decreased the MICs of rifampicin and cefotaxime by four- and twofold, respectively (data not shown). In E. coli, similarly, single mutations of kat and sod genes did not affect MIC levels but a katG and katE double mutation reduced the MIC of norfloxacin by two-fold. ${ }^{9}$ To examine lethality kinetics, C. jejuni was exposed to 10 times the MICs of ciprofloxacin and rifampicin, and bacterial viability was determined. Interestingly, the mutants in the oxidative stress defense system demonstrated significantly reduced viability compared with the wild-type (Figure 1). Overall, the viability reduction was more significant in the katA mutant compared with the $\operatorname{sodB}$ mutant, and bacterial killing was synergistically enhanced by the $\operatorname{sodB}$ and $k a t A$ double mutation particularly under rifampicin treatment (Figure 1b). Similarly, it was reported that a $\operatorname{sodA}$ mutation
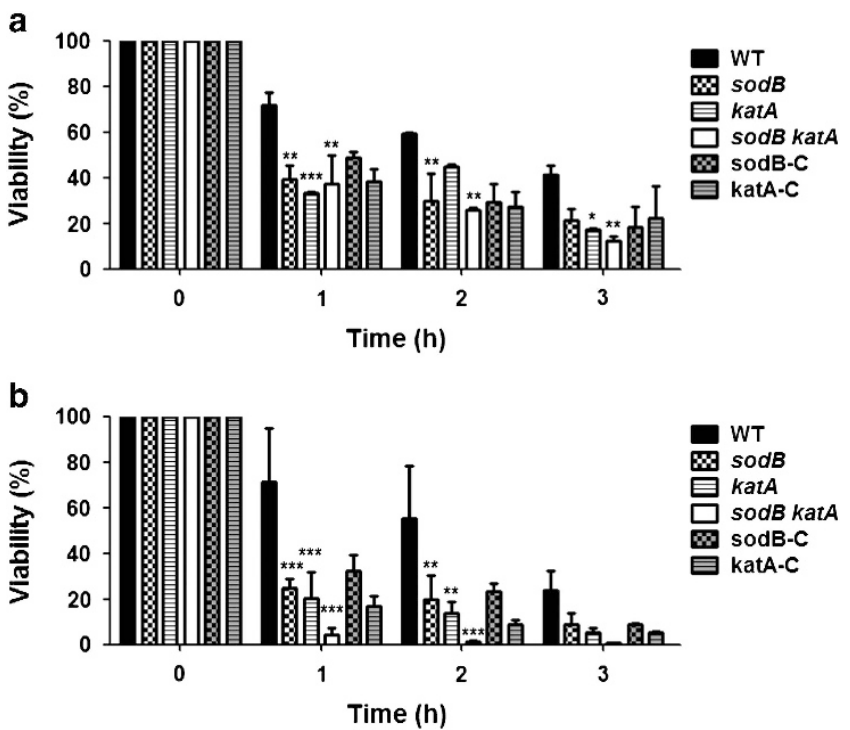

Figure 1 Viability of the wild-type (WT; C. jejuni NCTC 11168), sodB mutant $(\operatorname{sod} B)$, kat $A$ mutant $($ kat $A), \operatorname{sod} B$ and kat $A$ double mutant $(\operatorname{sod} B$ kat $A$ ), the $\operatorname{sod} B$ complementation strain (sodB-C) and the kat $A$ complementation strain (katA-C) after exposure to 10 times the MICs of ciprofloxacin (a) and rifampicin (b). The viability was calculated as CFU after antibiotic treatment was divided by CFU before the treatment. The results show the means and s.d. of three independent experiments. The statistical significance between the WT and the mutants was determined by two-way analysis of variance with Bonferroni's post-test using Prism software (version 5.01; Graphpad software Inc., La Jolla, CA, USA). ${ }^{*} P<0.05$, ${ }^{* *} P<0.01,{ }^{* * *} P<0.001$. in E. faecalis resulted in $3.9 \log _{10}$ decrease in viable counts by exposure to 20 times the MIC of vancomycin. ${ }^{3}$ The treatment of a $\operatorname{sodA}$ and $\operatorname{sodB}$ double mutant with 10 times the MIC of norfloxacin for $2 \mathrm{~h}$ reduced viable counts by 10 -fold compared with the wild-type E. coli. ${ }^{9}$ Previously, Kohanski et al. demonstrated that increased production of ROS by antibiotics leads to the destabilization of iron-sulfur clusters and the released $\mathrm{Fe}^{2+}$ produces hydroxyl radicals via the Fenton reaction. ${ }^{2}$ Hydroxyl radicals have a critical role in bacterial killing by antibiotics, and the production of hydroxyl radicals is mediated by a metabolic response under antibiotic treatment, which involves the tricarboxylic acid (TCA) pathway and subsequent depletion of $\mathrm{NADH}^{2}$ The importance of the TCA cycle in ROSmediated killing by antibiotics was reassured in $L$. monocytogenes. ${ }^{4}$ Unlike the TCA cycle of E. coli, L. monocytogenes has noncyclic TCA pathway lacking $\alpha$-ketoglutarate dehydrogenase, oxidative stress mutants, including $\Delta$ sod and $\Delta$ fri mutants, did not affect lethal kinetics by bactericidal antibiotics presumably due to the different TCA cycle. ${ }^{4}$ As the genome sequence of $C$. jejuni demonstrates the presence of all genes responsible for the TCA cycle, ${ }^{15} C$. jejuni would adopt the ROS-mediated killing mechanism similar to E. coli. However, the presence of different ROS-detoxifying enzymes and regulatory mechanisms of oxidative stress resistance still propose C. jejuni may have a unique mechanism for ROS-mediated killing by antibiotics.

On the basis of our findings and previous reports, the effect of oxidative stress defense on antibiotic lethality may vary in different bacterial species. As the sole catalase and superoxide dismutase in C. jejuni, KatA and SodB contribute to C. jejuni's resistance to ciprofloxacin and rifampicin. To the best of our knowledge, this is the first report demonstrating the implication of oxidative stress defense in the antibiotic resistance of Campylobacter. At this stage, the elucidation of molecular details associated with the ROSmediated lethal mechanism in Campylobacter still awaits future studies.

\section{ACKNOWLEDGEMENTS}

This study was supported by NSERC Discovery Grant 401843-2012-RGPIN to BJ. SH is supported by grant 2012-0004344 from the Basic Science Research Program through the National Research Foundation of Korea (NRF) funded by the Ministry of Education, Science and Technology, and SH is a recipient of the graduate fellowship provided by the Ministry of Education through the Brain Korea 21 Project.

1 Imlay, J. A. Cellular defenses against superoxide and hydrogen peroxide. Annu. Rev. Biochem. 77, 755-776 (2008).

2 Kohanski, M. A., Dwyer, D. J., Hayete, B., Lawrence, C. A. \& Collins, J. J. A common mechanism of cellular death induced by bactericidal antibiotics. Cell 130, 797-810 (2007).

3 Bizzini, A., Zhao, C., Auffray, Y. \& Hartke, A. The Enterococcus faecalis superoxide dismutase is essential for its tolerance to vancomycin and penicillin. J. Antimicrob. Chemother. 64, 1196-1202 (2009).

4 Feld, L., Knudsen, G. M. \& Gram, L. Bactericidal antibiotics do not appear to cause oxidative stress in Listeria monocytogenes. Appl. Environ. Microbiol. 78, 4353-4357 (2012)

5 Ruiz-Palacios, G. M. The health burden of Campylobacter infection and the impact of antimicrobial resistance: playing chicken. Clin. Infect. Dis. 44, 701-703 (2007).

6 Luangtongkum, T. et al. Antibiotic resistance in Campylobacter: emergence, transmission and persistence. Future Microbiol. 4, 189-200 (2009).

7 Atack, J. M. \& Kelly, D. J. Oxidative stress in Campylobacter jejuni: responses, resistance and regulation. Future Microbiol. 4, 677-690 (2009).

8 Jeon, B., Muraoka, W., Scupham, A. \& Zhang, Q. Roles of lipooligosaccharide and capsular polysaccharide in antimicrobial resistance and natural transformation of Campylobacter jejuni. J. Antimicrob. Chemother. 63, 462-468 (2009).

9 Wang, X. \& Zhao, X. Contribution of oxidative damage to antimicrobial lethality. Antimicrob. Agents Chemother. 53, 1395-1402 (2009). 
10 Wosten, M. M., Boeve, M., Koot, M. G., van Nuenen, A. C. \& van der Zeijst, B. A. Identification of Campylobacter jejuni promoter sequences. J. Bacteriol. 180, 594-599 (1998).

11 Karlyshev, A. V. \& Wren, B. W. Development and application of an insertional system for gene delivery and expression in Campylobacter jejuni. Appl. Environ. Microbiol. 71, 4004-4013 (2005).

12 Hwang, S., Kim, M., Ryu, S. \& Jeon, B. Regulation of oxidative stress response by CosR, an essential response regulator in Campylobacter jejuni. PLoS One 6, e22300 (2011).
13 Hwang, S., Zhang, Q., Ryu, S. \& Jeon, B. Transcriptional regulation of the CmeABC multidrug efflux pump and the KatA catalase by CosR in Campylobacter jejuni. J. Bacteriol. 194, 6883-6891 (2012).

14 Jeon, B., Wang, Y., Hao, H., Barton, Y. W. \& Zhang, Q. Contribution of CmeG to antibiotic and oxidative stress resistance in Campylobacter jejuni. J. Antimicrob. Chemother. 66, 79-85 (2011).

15 Parkhill, J. et al. The genome sequence of the food-borne pathogen Campylobacter jejuni reveals hypervariable sequences. Nature 403, 665-668 (2000). 